\title{
162. On the Summability of Fourier Series by Riesz's Logarithmic Means.
}

By Fu Traing WaNG.

Mathematical Institute, Tohoku Imperial University, Sendai.

(Comm. by M. FuJiwara, M.I.A., Dec. 12, 1933.)

1. Let $f(t)$ be a summable and periodic function with period $2 \pi$, and let

$$
f(t) \sim \frac{a_{0}}{2}+\sum_{n=1}^{\infty}\left(a_{n} \cos n t+b_{n} \sin n t\right) .
$$

The Fourier series $(1 \cdot 1)$ is said to be summable $(R, k)$, for $t=x$, to sum $s$, provided that

$$
R_{\omega}^{k}=\frac{a_{0}}{2}+\frac{1}{(\log \omega)^{k}} \sum_{n<\omega}\left(\log \frac{\omega}{n}\right)^{k}\left(a_{n} \cos n x+b_{n} \sin n x\right)
$$

tends to a limit $s$, as $\omega \rightarrow \infty$.)

Let

$$
\phi(u)=\frac{1}{2}\{f(x+u)+f(x-u)-2 s\} ;
$$

we write

$$
\phi(t) \rightarrow 0 \quad(R, \alpha)
$$

as $t \rightarrow 0$, provided that

when $t \rightarrow 0$.

$$
\psi_{\alpha}(t)=\frac{1}{\Gamma(\alpha)} \int_{t}^{\pi}\left(\log \frac{u}{t}\right)^{\alpha-1} \frac{\phi(u)}{u} d u=o\left[\left(\log \frac{1}{t}\right)^{\alpha}\right],
$$

Concerning the summability of Fourier series by Riesz's logarithmic means, Prof. Hardy has given a theorem on $(R, 1)$ summability. ${ }^{2)}$ Now we can extend this theorem and obtain some other theorems. The proof of them will appear in Tohoku Mathematical Journal.

2. Suppose that $k$ is a positive integer and $\psi_{0}(t)=\phi(t)$, then we have

Theorem A. If

$$
\int_{0}^{t}\left|\psi_{k-1}(u)\right| d u=O\left[t\left(\log \frac{1}{t}\right)^{k}\right],
$$

then the necessary and sufficient condition that the series (1.1) should be summable $(R, k)$, for $t=x$, to sum $s$, is that

1) Hardy and Riesz: Theory of general Dirichlet's series.

2) Hardy: Quarterly Journal, 2 (1931). 
No. 10.] On the Summability of Fourier Series of Riesz's Logarithmic Means. 569

and

$$
\begin{aligned}
& \psi_{k}(t)=O\left[\left(\log \frac{1}{t}\right)^{k}\right], \\
& \int_{0}^{t} \psi_{k}(u) d u=o\left[t\left(\log \frac{1}{t}\right)^{k}\right],
\end{aligned}
$$

when $t \rightarrow 0$.

Theorem B. If

$$
\int_{0}^{t}\left|\psi_{k-1}(u)\right| d u=o\left[t\left(\log \frac{1}{t}\right)^{k}\right],
$$

then the necessary and sufficient condition that the series $(1 \cdot 1)$ should be summable $(R, k)$ for $t=x$, to sum $s$, is that

$$
\phi(t) \rightarrow 0 \quad(R, k),
$$

when $t \rightarrow 0$.

Theorem C. The necessary and sufficient condition that the series $(1 \cdot 1)$ should be summable by Riesz's logarithmic means, for $t=x$, to sum $s$, is that $\phi(t) \rightarrow 0(R, k)$, for some $k$.

Theorem D. If

$$
\phi_{k}(t)=\int_{0}^{t}(t-u)^{k-1} \phi(u) d u=o\left(t^{k}\right),
$$

when $t \rightarrow 0$, then the series $(1 \cdot 1)$ is summable $(R, k)$, for $t=x$, to sum $s$.

Theorem E. If $\alpha>0$, and

$$
\phi(t) \rightarrow 0 \quad(R, \alpha),
$$

when $t \rightarrow 0$, then the series (1.1) is summable $(R, \alpha+\delta)(\delta>0)$, for $t=x$, to sum s.

Theorem F. If the Fourier series $(1 \cdot 1)$ is summable $(R, \alpha)$, for $t=x$, to sum $s$, then

when $t \rightarrow 0$.

$$
\phi(t) \rightarrow 0 \quad(R, \alpha+1+\delta),
$$

Theorem G. If

$$
\int_{0}^{t}|\phi(u)| d u=O(t)
$$

then the necessary and sufficient condition that the series $(1 \cdot 1)$ should be summable by Riesz's logarithmic means of any positive order, for $t=x$, to sum $s$, is that

$$
\int_{t}^{\pi} \frac{\phi(u)}{u} d u=o\left(\log \frac{1}{t}\right)
$$

when $t \rightarrow 0$. 